\title{
POSSIBILITÉ D'UTILISATION DU PROTACTINIUM 233 COMME SIMULATEUR DU PLUTONIUM 239 EN DOSIMÉTRIE PULMONAIRE
}

\author{
P. NIKPAY-ASLIE* \\ (Manuscrit reçu le 4 novembre 1974)
}

\begin{abstract}
RESUME
Pour mesurer une charge pulmonaire en ${ }^{239} \mathrm{Pu}$, émetteur de raies $\mathbf{X}$ de très basses énergies, il a été proposé une méthode consistant en l'administration au sujet contaminé d'un aérosol de $233 \mathrm{~Pa}$. En effet, le ${ }^{233} \mathrm{~Pa}$, de courte période, émet, outre les mêmes raies $\mathbf{X}$ que le ${ }^{239} \mathrm{Pu}$, un photon $\gamma$ ayant une énergie de $310 \mathrm{keV}$, qui permet de mesurer la charge administrée.

Pour la mesure des basses énergies, les possibilités des détecteurs à scintillation et d'un compteur proportionnel ont été comparées à partir de mesures effectuées sur un fantôme anthropomorphe équivalent-tissu. Le compteur proportionnel permet la mesure des raies $\mathrm{X}$ et l'utilisation du ${ }^{233} \mathrm{~Pa}$ comme simulateur du $239 \mathrm{Pu}$.
\end{abstract}

\begin{abstract}
For the measurement of a pulmonary charge by ${ }^{239} \mathrm{Pu}$, an emitter of $\mathrm{X}$ rays of very low energies, a method has been suggested consisting of administering to the patient an aerosol of ${ }^{233} \mathrm{~Pa}$. In effect, the ${ }^{233} \mathrm{~Pa}$ with a short period, emits in addition to the same $\mathrm{X}$ rays a photon $\gamma$ of $310 \mathrm{keV}$ allowing the measurement of the administered charge.

For the measurement of low energies, the possibilities of a scintillation detector and proportional counter have been compared, by means of measurement made on an antropomorphous phantom tissue -équivalent material. The proportional counter permits the measurement of the $\mathrm{X}$ Rays and the use of ${ }^{233} \mathrm{~Pa}$ as a simulator of ${ }^{239} \mathrm{Pu}$.
\end{abstract}

\section{INTRODUCTION}

Le plutonium 239 est un radionucléide particulièrement important dans le domaine des radiocontaminations. Il est en effet d'une grande toxicité du fait de son émission alpha, de son métabolisme, et de sa longue période.

- Centre d'Etudes Nucléaires de l'Université de Téhéran (Iran).

Travail effectué dans le Département de Protection du C.E.A.C.E.N.F.A.R. et dans le Service de Radiopathologie de la Fondation Curie - Institut du Radium.

RADIOPROTECTION, VOL. 9 - $\mathrm{N}^{\circ} 3$ 
Les quantités maximales admissibles sont très faibles. Le poumon représente la porte d'entrée la plus fréquente dans les accidents. Le problème est donc celui de l'évaluation d'une charge pulmonaire à très bas niveau. Il est rendu d'autant plus difficile que les photons émis par le plutonium 239 sont de très basse énergie. Pour évaluer la charge on procède généralement par comparaison de mesures effectuées sur le sujet et sur un fantôme contenant une activité connue. En fait, l'évaluation est imprécise et l'étalonnage à partir du sujet lui-même a été proposé, en utilisant le protactinium 233 comme simulateur du plutonium 239. Il émet les mêmes raies X de basses énergies que le plutonium 239, mais émet de surcroît un photon gamma convenant parfaitement à la détection externe. Ce sont les possibilités relatives à cette dernière méthode qui sont étudiées.

\section{TRAVAIL EXPERIMENTAL}

\subsection{MATÉRIEL D'ÉtUde}

Une fois le protactinium 233 administré à l'Homme par voie respiratoire, sous forme d'aérosol, le problème est d'en mesurer la charge pulmonaire exacte, de dissocier les raies $\mathrm{X}$ du reste du spectre et de les mesurer. L'aérosol doit être de type insoluble, et le diamètre des particules doit être de l'ordre de $1 \mu \mathrm{m}$. Les mesures après aérosol doivent être pratiquées après un délai de quelques jours, pour permettre l'évacuation de l'inévitable activité bronchique et digestive. Pour apprécier les possibilités de mesure chez l'Homme, on a effectué des mesures sur un fantôme anthropomorphe équivalent-tissu, comprenant des organes se prêtant à l'incorporation de radio-éléments (fig.1).

En ce qui concerne la détection, deux qualités sont recherchées, !a sensibilité et la résolution. On a utilisé deux types de détecteurs : des compteurs à scintillation et un compteur proportionnel.

Les radionucléides utilisés sont donc le plutonium 239 et le protactinium 233, dont les caractéristiques radioactives essentielles sont rappelées. Les périodes sont de $2,4 \times 10^{4}$ ans pour ${ }^{239} \mathrm{Pu}$ et de 27,4 jours pour ${ }^{23} \mathrm{~Pa}$. ${ }^{239} \mathrm{Pu}$ émet des raies $\mathrm{X}$ de $13,6 \mathrm{keV}, 17,2 \mathrm{keV}$ et $20,2 \mathrm{keV}$. En plus des raies $\mathrm{X}$ de basses énergies, ${ }^{233} \mathrm{~Pa}$ émet un photon gamma ayant une énergie de $310 \mathrm{keV}$.

\subsection{ETUdE}

Les mesures ont été effectuées, d'une part, sur ${ }^{239} \mathrm{Pu}$, d'autre part, sur ${ }^{233} \mathrm{~Pa}$. Les compteurs, qu'ils soient à scintillation ou proportionnels, étaient placés dans des enceintes blindées, de manière à réduire le bruit de fond au minimum.

\subsubsection{Détecteurs à scintillation}

Ils comportent un cristal d'NaI (Tl) de grandes dimensions : $20 \mathrm{~cm}$ de diamètre. Des mesures point par point ont été effectuées sur le thorax, 


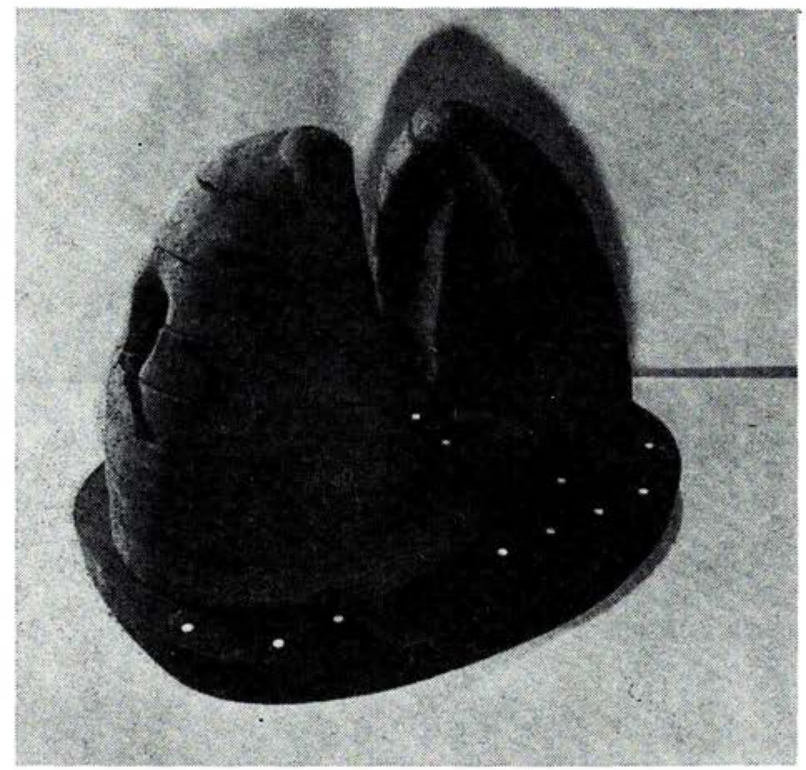

FIG. 1. - Poumons du fantôme anthropomorphe

dans le sens longitudinal et transversal, soit manuellement, soit selon un déplacement discontinu automatique. On observe les faits suivants :

- pour ${ }^{2 s 9} \mathrm{Pu}$ :

non séparation des trois raies $\mathbf{X}$;

déplacement du pic photo-électrique vers le pic de plus grande énergie, du fait que les pics dus aux trois raies $\mathrm{X}$ sont différemment absorbés.

Rôle de la distance sonde - sujet :

La distance de $2,5 \mathrm{~cm}$, minimale pour pouvoir réaliser les balayages, fournit un taux de comptage de $87 \%$ de celui effectué à $0,5 \mathrm{~cm}$.

Sensibilité :

La limite inférieure de détection est de l'ordre de $1 \mu \mathrm{Ci}$.

- pour ${ }^{2 s s} \mathrm{~Pa}$ :

Le pic répondant au groupe de raies $\mathrm{X}$ n'est plus identifiable, et 1e compteur à scintillation n'est donc pas utilisable pour la mesure des raies X. Le pic répondant à l'énergie de $310 \mathrm{keV}$ est par contre facilement mesuré et il permet d'évaluer la charge pulmonaire après administration d'aérosol. Cette évaluation de la charge est effectuée à partir de deux fantômes dont l'équivalent morphologique a été déterminé en utilisant des mesures pratiquées sur l'Homme, après administration intra-veineuse de produits radio- 
actifs en vue de scintigraphies pulmonaires. Les mesures pratiquées sur ces deux fantômes chargés en ${ }^{233} \mathrm{~Pa}$ permettent d'établir une courbe de rendement en fonction de la morphologie pour une énergie de $310 \mathrm{keV}$, avec une précision de $2 \%$.

\subsubsection{Compteur proportionnel}

Ses caractéristiques sont les suivantes :

- résolution : elle est de $12 \%$ pour la raie à $13,6 \mathrm{keV}$ et de $11,7 \%$ pour la raie à $17,2 \mathrm{keV}$.

- mouvement propre : il est de 10 coups par minute dans la bande des basses énergies du plutonium 239, c'est-à-dire entre $10 \mathrm{keV}$ et $20 \mathrm{keV}$, et de 3 coups par minute dans la bande de 50 à $70 \mathrm{keV}$.

- linéarité en fonction de l'énergie : elle est parfaite jusqu'à $60 \mathrm{keV}$.

- efficacité du compteur: les mesures expérimentales d'efficacité donnent les résultats suivants :

\begin{tabular}{|c|c|}
\hline 14 & $\mathrm{keV} \quad\left({ }^{57} \mathrm{Co}\right)$ \\
\hline 17,2 & $\mathrm{keV}$ \\
\hline 20,2 & $\mathrm{keV}$ \\
\hline 60 & $\mathrm{keV}$ \\
\hline
\end{tabular}

Son intérêt réside dans la mesure des raies $\mathrm{X}$.

- pour ${ }^{2 s 9} \mathrm{Pu}$ :

On sépare très bien les deux raies $\mathrm{X}$ de $13,6 \mathrm{keV}$ et $17,2 \mathrm{keV}$ (fig. 2).

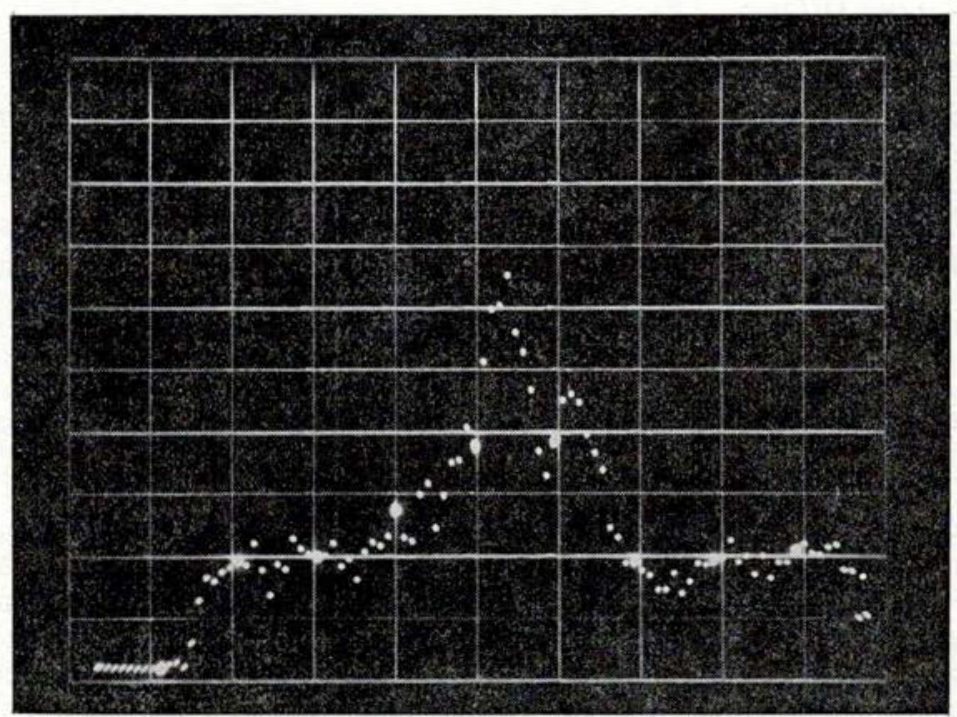

FIG. 2. - Spectre du ${ }^{239} \mathrm{Pu}$ dans le fantôme 
On observe les mêmes variations de taux de comptage quand on fait varier la distance sonde-sujet que pour le détecteur à scintillation. En ce qui concerne la sensibilité : pour des mesures effectuées à $5 \mathrm{~cm}$ du sujet, la limite inférieure détectable est de l'ordre de $0,5 \mu \mathrm{Ci}$.

- pour ${ }^{233} \mathrm{~Pa}$ (fig. 3) :

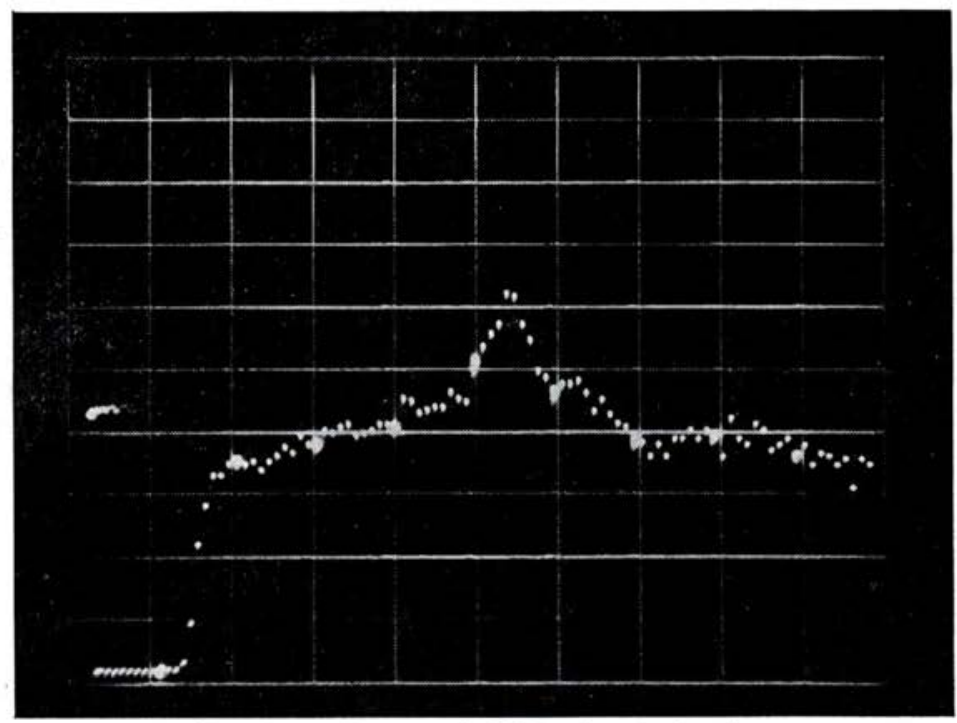

FIg. 3. - Spectre du ${ }^{233} \mathrm{~Pa}$ dans le fantôme

Les deux raies $\mathrm{X}$ sont aussi bien séparées. Leur mesure est possible. Elle est cependant rendue difficile en raison des autres énergies émises. Il est nécessaire d'éliminer le pied sur lequel reposent les pics répondant aux raies X. Cette opération est effectuée avec une précision de $10 \%$. Cette valeur est obtenue en comparant les rapports des coups pour ${ }^{233} \mathrm{~Pa}$ et ${ }^{239} \mathrm{Pu}$, obtenus, d'une part, de façon théorique et, d'autre part, de façon expérimentale. Pour le calcul théorique, on utilise les coefficients d'émission des raies X $\left(27 \%\right.$ pour ${ }^{233} \mathrm{~Pa}$ et $4 \%$ pour $\left.{ }^{239} \mathrm{Pu}\right)$. L'expérimentation a donné les résultats suivants :

- mesure pulmonaire ${ }^{239} \mathrm{Pu}$ :

détection ventrale: 19 coups par minute par microcurie détection dorsale : 55 coups par minute par microcurie

- mesure pulmonaire ${ }^{233} \mathrm{~Pa}$ :

détection ventrale: 520 coups par minute par microcurie détection dorsale : 1110 coups par minute par microcurie 
Une activité pulmonaire de quelques microcuries est suffisante pour permettre une mesure ayant une précision convenable avec un temps de comptage approprié à l'Homme.

\section{CONCLUSION}

La résolution du compteur proportionnel permet dans le cas d'une localisation pulmonaire, de séparer les raies $\mathrm{X}$ du ${ }^{239} \mathrm{Pu}$. La séparation des raies $\mathrm{X}$ du ${ }^{233} \mathrm{~Pa}$, après administration d'un aérosol, est aussi possible ainsi que leur mesure bien que celle-ci présente une difficulté liée à l'émission de photons. La limite inférieure de détection est de $0,5 \mu \mathrm{Ci}$.

Par ailleurs, l'activité incorporée en protactinium 233 est mesurable en utilisant des compteurs à cristal de $\mathrm{NaI}(\mathrm{Tl})$. Quelques microcuries permettent de mesurer les raies $\mathrm{X}$, la dose délivrée étant alors très faible. La précision de cette mesure est de l'ordre de $10 \%$. Cette technique pourrait donc permettre de mesurer une charge pulmonaire en plutonium 239 pourvu qu'elle soit égale ou supérieure à $0,5 \mu \mathrm{Ci}$, avec une précision qui est de l'ordre de $15 \%$.

\section{BIBLIOGRAPHIE}

[1] Boulay P., Chivot J.J., Jeanmaire L. et Lansiart A. - Le protactinium 233 " simulateur de plutonium " pour la mesure directe d'une contamination pulmonaire. Physique Médicale. C.R. Acad. Sc., Paris, 265, p. 1571-1574 (13 novembre 1967).

[2] Boulay P. - Mise au point d'une méthode directe de détermination de la charge corporelle en plutonium 239 chez l'homme par détection X de l'uranium 235. Rapport C.E.A.-R-3559 (1968).

[3] Brière M., Jouve B., PAulin R. - Mesures de radioactivité. Manuel pratique d'utilisation. Paris, Hermann Ed. (1970).

[4] Henry P. - Métabolisme et toxicologie du Plutonium 239. Evaluation de la contamination interne des personnes professionnellement exposées. Rapport C.E.A., $\mathbf{n}^{\circ} 2381$ (1963).

[5] I.C.R.P. - Report of Committee II on permissible dose for internal radiation. Health Physics, 3 (1960).

[6] LANSIART A., Morucci J.P. - Nouveau compteur proportionnel destiné à la détection in vivo des traces de plutonium dans les poumons. Assessment of radioactivity in man C.R. Symposium Heidelberg, p. 114-140 (1964).

[7] Morucci J.P. - Nouveau compteur proportionnel destiné à la détection in vivo. Thèse-Docteur-Ingénieur, Faculté des Sciences de Paris (1966).

[8] Trouble M. - Etude d'une méthode de détection du plutonium par spectographie X. Application à la mesure des contaminations sur l'homme. Rapport C.E.A.-R-3098 (1967).

[9] West D., Dawson J.K., Mandelberg C.J. - The investigation of soft radiation from plutonium 239 and uranium 233 with a proportionnal counter. Philos. Mag., 43, 7 (343), 875 (1952). 